\title{
Navigation Modeling and Simulation for Canterbury Hover-Capable Underwater Vehicle
}

\author{
W.H. Wang* R.C. Engelaar** J.H.A.M. Vervoort** \\ P.P.J. van den Bosch ${ }^{* *}$ J.G. Chase* X.Q. Chen* \\ * Mechanical Engineering, University of Canterbury, 20 Kirdwood Ave, \\ Christchurch, New Zealand 8140 (e-mail: wenhui.wang, xiaoqi.chen, \\ geoff.chase@canterbury.ac.nz). \\ ** Electrical Engineering, Eindhoven University of Technology, \\ Eindhoven, The Netherlands (e-mail: r.c.engelaar, \\ j.h.a.m.vervoort@student.tue.nl, p.p.j.v.d.bosch@tue.nl)
}

\begin{abstract}
Development of underwater vehicles (UVs) has come to an era that UVs can be specifically designed and made for customized applications, based on established theories and principles. This paper reports on the Canterbury UV developed at the University of Canterbury, which targets to inspect and clean sea chests of ships to eliminate bio-security risks caused by small organisms residing in the sea chests. Based on the mechanical prototype, navigation of the Canterbury UV is discussed in principle with the aim to providing theoretical ground for the future implementation of navigation control. Simulation results of the UV model demonstrate that the vehicle can be controlled with satisfying performance using the extended Kalman filter.
\end{abstract}

Keywords: Modeling, navigation, underwater vehicle, extended Kalman filter, control.

\section{INTRODUCTION}

Starting from 1960s, underwater vehicles (UVs) have been performing many tasks such as the detection of mines, security of harbors, mapping of the ocean floor and maintenance of cables, etc. Griffiths (2003). The basic theories and principles for UVs, after dozens of years of evolvement, have become mature. Recently, based on and enabled by these established theories and principles, many efforts have been made to customize UVs for special scenarios. By integrating new advances in battery, computer, electronics, and control, these UVs prove to be capable of fulfilling a set of pre-defined tasks for their target applications. Targeting a niche application, this paper focuses on the navigation modeling and simulation for the UV developed recently at the University of Canterbury.

Many small organisms survive in the sea chests of ships and are transported around the world creating a biosecurity risk. The Canterbury UV was designed with the primary purpose for inspecting and cleaning the sea chests, an application with significant impact in the area of biosecurity. To work properly in this scenario, the Canterbury UV was designed to meet these key specifications: i) low cost for mass deployment for shallow water tasks no deeper than 20m; ii) slightly positive buoyancy so that it does not sink to the sea/water bed when power runs off; iii) compact structure for easy on-site maintenance and trouble shooting; iv) extendable space for carrying payload to inspect and clean the sea chests; v) manual or autonomous navigation at a cruise speed of $\sim 0.5 \mathrm{~m} / \mathrm{s}$.

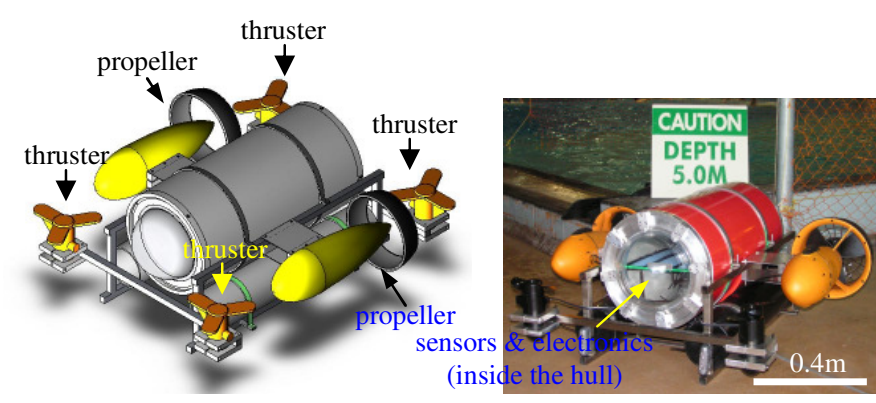

Fig. 1. Mechanical structure of the Canterbury UV. Left: Isometric view. Right: Real picture

Fig. 1 shows the prototype. Two propellers are mounted aside and four thrusters on the corners to allow the vehicle hover-capable. See Wang et al. for detailed information.

Based on the mechanical prototype, the paper is organized as follows. Firstly, the specific dynamic model for the Canterbury UV is derived; then the extended Kalman filter (EKF) is presented to estimate the vehicle position for navigation. Next, the navigation control is demonstrated with the model and EKF via simulation, followed by discussions and future work. Finally, the paper concludes.

\section{DYNAMIC MODEL}

\subsection{Reference Frames}

Two reference frames shown in Fig. 2 are used to describe an underwater vehicle: the earth-fixed frame (EFF), $X Y Z$, fixed in space; and the body-fixed frame (BFF), $X_{0} Y_{0} Z_{0}$, 


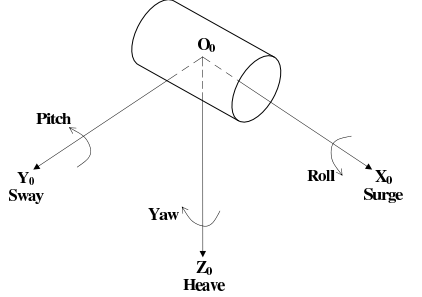

(a) Body-fixed frame (BFF)

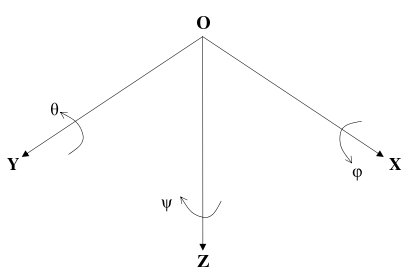

(b) Earth-fixed frame (EFF)

Fig. 2. Reference frames

Table 1. Notation used for marine vehicles

\begin{tabular}{ccccc} 
DOF & Motion/rotation & $\begin{array}{c}\text { Forces/ } \\
\text { moments }\end{array}$ & $\begin{array}{c}\text { Linear/ } \\
\text { angular } \\
\text { velocities }\end{array}$ & $\begin{array}{c}\text { Positions/ } \\
\text { Euler } \\
\text { angles }\end{array}$ \\
\hline 1 & In $x$-direction (surge) & $X$ & $u$ & $x$ \\
2 & In $y$-direction (sway) & $Y$ & $v$ & $y$ \\
3 & In $z$-direction (heave) & $Z$ & $w$ & $z$ \\
4 & About $x$-axis (roll) & $K$ & $p$ & $\phi$ \\
5 & About $y$-axis (pitch) & $M$ & $q$ & $\theta$ \\
6 & About $z$-axis (yaw) & $N$ & $r$ & $\psi$ \\
\hline
\end{tabular}

attached to the vehicle body. For EFF, the $Z$-axis is parallel to gravity. For BFF, the $X_{0}$-axis is aligned with the forward direction of the vehicle, the $Y_{0}$-axis is positive to starboard, and the $Z_{0}$-axis is positive downward. The origin of $\mathrm{BFF},\left(\mathrm{O}_{0}\right)$ coincides with the center of gravity of the vehicle.

For a UV, which is a rigid body, six degrees of freedom suffice to determine the position and orientation, namely, surge, sway, heave, roll, pitch and yaw. In this paper, the SNAME (The Society of Naval Architects \& Marine Engineers) notation (1950) is used, as listed in table 1.

These vectors are defined for subsequent sections: $\eta=$ $[x, y, z, \phi, \theta, \psi]^{T}$ in EFF, and $\nu=[u, v, w, p, q, r]^{T}$ in BFF. The forces and moments acting on the vehicle in BFF are defined by $\tau=[X, Y, Z, K, M, N]^{T}$.

\subsection{General Model}

The dynamic model that is widely used to describe underwater vehicles proposed in Fossen (1994) applies to our vehicle, which is

$$
\begin{aligned}
\mathbf{M} \dot{\nu}+\mathbf{C}(\nu) \nu+\mathbf{D}(\nu) \nu+\mathbf{g}(\eta) & =\tau \\
\dot{\eta} & =\mathbf{J}(\eta) \nu
\end{aligned}
$$

where $\mathbf{M}$ is the mass (inertial) matrix, $\mathbf{C}$ is the Coriolis and centripetal matrix, $\mathbf{D}$ the drag matrix, and $\mathbf{g}$ the restoring force vector.

\subsection{Mass Matrix}

The mass matrix is composed of two parts: the mass of the rigid body; and added mass, a virtual mass describing the extra force required to push a body through a fluid van de Ven et al. (2005). Thus

$$
\mathbf{M}=\mathbf{M}_{R B}+\mathbf{M}_{A}
$$

where $\mathbf{M}_{R B}$ and $\mathbf{M}_{A}$ are the inertia matrices for rigid body and added mass, respectively.
Suppose the center of gravity of the vehicle is in the coordinates $\left(x_{g}, y_{g}, z_{g}\right), \mathbf{M}_{R B}$ is given by

$$
\mathbf{M}_{R B}=\left(\begin{array}{ccc|ccc}
m & 0 & 0 & 0 & m z_{g} & -m y_{g} \\
0 & m & 0 & -m z_{g} & 0 & m x_{g} \\
0 & 0 & m & m y_{g} & -m x_{g} & 0 \\
\hline 0 & -m z_{g} & m y_{g} & I_{x x} & -I_{x y} & -I_{x z} \\
m z_{g} & 0 & -m x_{g} & -I_{y x} & I_{y y} & -I_{y z} \\
-m y_{g} & m x_{g} & 0 & -I_{z x} & -I_{z y} & I_{z z}
\end{array}\right)
$$

where $m$ is the vehicle mass, $I$ s are the elements of the moment of inertia tensor. Also, as the vehicle is symmetric w.r.t. the $x-z$ plane, $I_{x y}=I_{y x}=0$ and $I_{y z}=I_{z y}=0$. Furthermore, the center of gravity is in the origin of BFF (see Section 2.1), so $\left(x_{g}, y_{g}, z_{g}\right)=(0,0,0)$ and therefore (3) becomes El-Hawary (2001).

$$
\mathbf{M}_{R B}=\left(\begin{array}{cccccc}
m & 0 & 0 & 0 & 0 & 0 \\
0 & m & 0 & 0 & 0 & 0 \\
0 & 0 & m & 0 & 0 & 0 \\
0 & 0 & 0 & I_{x x} & 0 & -I_{x z} \\
0 & 0 & 0 & 0 & I_{y y} & 0 \\
0 & 0 & 0 & -I_{x z} & 0 & I_{z z}
\end{array}\right)
$$

The added mass matrix is given below:

$$
\mathbf{M}_{A}=-\left(\begin{array}{cccccc}
X_{\dot{u}} & X_{\dot{v}} & X_{\dot{w}} & X_{\dot{p}} & X_{\dot{q}} & X_{\dot{r}} \\
X_{\dot{v}} & Y_{\dot{v}} & Y_{\dot{w}} & Y_{\dot{p}} & Y_{\dot{q}} & Y_{\dot{r}} \\
X_{\dot{w}} & Y_{\dot{w}} & Z_{\dot{w}} & Z_{\dot{p}} & Z_{\dot{q}} & Z_{\dot{r}} \\
X_{\dot{p}} & Y_{\dot{p}} & Z_{\dot{p}} & K_{\dot{p}} & K_{\dot{q}} & K_{\dot{r}} \\
X_{\dot{q}} & Y_{\dot{q}} & Z_{\dot{q}} & K_{\dot{q}} & M_{\dot{q}} & M_{\dot{r}} \\
X_{\dot{r}} & Y_{\dot{r}} & Z_{\dot{r}} & K_{\dot{r}} & M_{\dot{r}} & N_{\dot{r}}
\end{array}\right)
$$

where the notation

$$
X_{\dot{u}}=\frac{\partial X}{\partial \dot{u}}
$$

As the vehicle is symmetric about the $x-z$ plane, according to Jones et al. (2002), some parameters are known to be zero, i.e.

$$
\mathbf{M}_{A}=-\left(\begin{array}{cccccc}
X_{\dot{u}} & 0 & X_{\dot{w}} & 0 & X_{\dot{q}} & 0 \\
0 & Y_{\dot{v}} & 0 & Y_{\dot{p}} & 0 & Y_{\dot{r}} \\
X_{\dot{w}} & 0 & Z_{\dot{w}} & 0 & Z_{\dot{q}} & 0 \\
0 & Y_{\dot{p}} & 0 & K_{\dot{p}} & 0 & K_{\dot{r}} \\
X_{\dot{q}} & 0 & Z_{\dot{q}} & 0 & M_{\dot{q}} & 0 \\
0 & Y_{\dot{r}} & 0 & K_{\dot{r}} & 0 & N_{\dot{r}}
\end{array}\right)
$$

\subsection{Coriolis Matrix}

The Coriolis force is an inertial force exerted on a body when it moves in a rotating reference frame. It is a summation of two matrices, related to the rigid body mass and added mass.

$$
\mathbf{C}(\nu)=\mathbf{C}_{R B}(\nu)+\mathbf{C}_{A}(\nu)
$$

Taking into account that the vehicle is symmetric w.r.t. the $x-z$ plane and that the center of gravity is placed in the origin of the BFF, the rigid body Coriolis and centripetal matrix is given by Silpa-Anan et al. (2000):

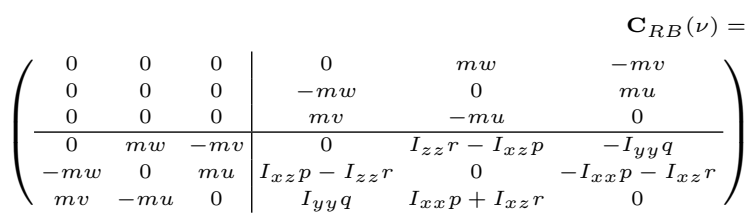


The added mass Coriolis matrix is given by El-Hawary (2001):

$$
\mathbf{C}_{A}(\nu)=\left(\begin{array}{ccc|ccc}
0 & 0 & 0 & 0 & c_{15} & c_{16} \\
0 & 0 & 0 & -c_{15} & 0 & c_{26} \\
0 & 0 & 0 & -c_{16} & -c_{26} & 0 \\
\hline 0 & c_{15} & c_{16} & 0 & c_{45} & c_{46} \\
-c_{15} & 0 & c_{26} & -c_{45} & 0 & c_{56} \\
-c_{16} & -c_{26} & 0 & -c_{46} & -c_{56} & 0
\end{array}\right)
$$

where

$$
\begin{array}{ll}
c_{15}=-X_{\dot{w}} u-Z_{\dot{w}} w-Z_{\dot{q}} q & c_{45}=-Y_{\dot{r}} v-K_{\dot{r}} p-N_{\dot{r}} r \\
c_{16}=Y_{\dot{v}} v+Y_{\dot{p}} p+Y_{\dot{r}} r & c_{46}=X_{\dot{q}} u+Z_{\dot{q}} w+M_{\dot{q}} q(11) \\
c_{26}=-X_{\dot{u}} u-X_{\dot{w}} w-X_{\dot{q}} q & c_{56}=-Y_{\dot{p}} v-K_{\dot{p}} p-K_{\dot{r}} r
\end{array}
$$

\subsection{Drag Matrix}

When an object moves in a fluid it experiences hydrodynamic drag forces, which are linear and quadratic to the velocity and given by:

$$
\mathbf{D}(\nu)=\mathbf{D}_{\text {lin }}+\mathbf{D}_{\text {nonlin }}(\nu)
$$

The linear drag matrix is given by:

$$
\mathbf{D}_{\operatorname{lin}}=-\left(\begin{array}{cccccc}
X_{u} & 0 & X_{w} & 0 & X_{q} & 0 \\
0 & Y_{v} & 0 & Y_{p} & 0 & Y_{r} \\
Z_{u} & 0 & Z_{w} & 0 & Z_{q} & 0 \\
0 & K_{v} & 0 & K_{p} & 0 & K_{r} \\
M_{u} & 0 & M_{w} & 0 & M_{q} & 0 \\
0 & N_{v} & 0 & N_{p} & 0 & N_{r}
\end{array}\right)
$$

where the notation $X_{u}$ is same as in (6).

The nonlinear drag force is given by

$$
F_{d r a g}=\frac{1}{2} \cdot \rho \cdot v^{2} \cdot c_{d} \cdot S
$$

Using notation in (6), we rewrite the nonlinear drag force to

$$
-\left(\begin{array}{cccccc}
X_{u|u|}|u| & 0 & X_{w|w|}|w| & 0 & X_{q|q|}|q| & 0 \\
0 & Y_{v|v|}|v| & 0 & Y_{p|p|}|p| & 0 & Y_{r|r|}|r| \\
Z_{u|u|}|u| & 0 & Z_{w|w|}|w| & 0 & Z_{q|q|}|q| & 0 \\
0 & K_{v|v|}|v| & 0 & K_{p|p|}|p| & 0 & K_{r|r|}|r| \\
M_{u|u|}|u| & 0 & M_{p|p|}|p| & 0 & M_{q|q|}|q| & 0 \\
0 & N_{v|v|}|v| & 0 & N_{p|p|}|p| & 0 & N_{r|r|}|r|
\end{array}\right) .
$$

Here the model assumes that the drag forces are symmetric, i.e., the drag coefficients for $+x$ and $-x$ are equal, so are the coefficients for $+z$ and $-z$. This is a very common assumption in the standard underwater vehicle models Alessandri et al. (1998).

\subsection{Restoring Force Vector}

The restoring force, also called the buoyancy and gravity forces vector, is the resultant of the gravity and buoyancy force and a function of the orientation of the vehicle.

When the center of buoyancy is given in BFF coordinates $\left[x_{B}, y_{B}, z_{B}\right]^{T}$ and the center of gravity is in the origin of $\mathrm{BFF}$, the restoring force vector is given by Antonelli (2007) and Ridao et al. (2004)

$$
\mathbf{g}(\nu)=\left(\begin{array}{c}
(W-B) \sin \theta \\
-(W-B) \cos \theta \sin \phi \\
-(W-B) \cos \theta \cos \phi \\
y_{B} B \cos \theta \cos \phi-z_{B} B \cos \theta \sin \phi \\
-z_{B} B \sin \theta-x_{B} B \cos \theta \cos \phi \\
x_{B} B \cos \theta \sin \phi+y_{B} B \sin \theta
\end{array}\right)
$$

where the gravitational force $W$, and the buoyancy force $B$, are given by

$$
W=m \cdot g, \quad B=\rho \cdot V \cdot g
$$

where $m$ denotes the vehicles mass, $g$ the gravitational acceleration, $\rho$ the density of the fluid and $V$ the volume of the vehicle.

\subsection{Forces and Moments Vector}

The forces and moments vector is a result of the input forces delivered by the thrusters of the vehicle. To find the force exerted by the thruster, a simplified thruster model $\tau_{i}=c_{v t} V|V|$ for low speed vehicles can be used, where $c_{v t}$ is the voltage-to-thrust coefficient, and $V$ is the applied voltage.

The forces and moments vector is given by $\tau=\mathbf{B U}$, where the thruster configuration matrix, $\mathbf{B}$, is a $6 \times n$ matrix which is dependent on the voltage-to-thrust coefficients and the location of the thrusters. The input voltage vector, $\mathbf{U}$, is a $n \times 1$ vector, with $n$ the number of thrusters, which is of the from $\left[V_{1}\left|V_{1}\right|, V_{2}\left|V_{2}\right|, \ldots, V_{n}\left|V_{n}\right|\right]^{T}$. Where $V_{i}$ is the applied voltage to the $i$-th thruster.

\subsection{Specific Canterbury Underwater Vehicle Model}

The Canterbury UV has some specific properties which allow to further simplify the model described in Sections 2.2-2.7, which couples degrees of freedom. According to Wang and Clark (2006), when a vehicle is symmetric w.r.t. the $x-z$ plane and close to symmetric w.r.t. the $y-z$ plane, which is our case, the motions in surge, sway, pitch and yaw can be decoupled. Also, for low speed vehicles, the other directions can also be decoupled. Furthermore, the off-diagonal elements of the added mass matrix and drag matrix are much smaller than their diagonal counterparts Alessandri et al. (1998). Because of these assumptions, the mass matrix, coriolis matrix and drag matrix are diagonal matrices.

$$
\begin{gathered}
\mathbf{M}=\operatorname{diag}\left(m-X_{\dot{u}}, m-Y_{\dot{v}}, m-Z_{\dot{w}}\right. \\
\left.I_{x x}-K_{\dot{p}}, I_{y y}-M_{\dot{q}}, I_{z z}-N_{\dot{r}}\right) \\
\mathbf{D}(\nu)=-\operatorname{diag}\left(X_{u}+X_{u|u|}|u|, Y_{v}+Y_{v|v|}|v|, Z_{w}+Z_{w|w|}|w|\right. \\
\left.K_{p}+K_{p|p|}|p|, M_{q}+M_{q|q|}|q|, N_{r}+N_{r|r|}|r|\right)
\end{gathered}
$$

Particularly, $\mathbf{C}(\nu)=0$ because in (9) and (10) the diagonal entries of this matrix are zero.

Because the vehicle is stable in the roll and pitch angle, these angles are assumed to be zero. Assume the center of buoyancy is vertically aligned with the center of gravity, i.e., $\left[0,0, z_{B}\right]^{T}$. These two assumptions lead to

$$
\mathbf{g}=(0,0,-(W-B), 0,0,0)^{T}
$$


Experiments have been conducted to obtain the voltageto-thrust coefficients of the thrusters. The relationship between the duty-cycle and the developed thrust can be formulated as $\tau_{i}=0.446 D_{i}-2.05$, with $\tau_{i}$ the delivered thrust in $[N]$ and $D_{i}$ the applied duty-cycle in [\%] to thruster $i$. The relationship was almost the same and is assumed equal for all six thrusters.

Because motions are decoupled; and only motions in surge, yaw, and heave are non-zero, powered by thrusters, the forces and moments vector is

$$
\tau=\left(\begin{array}{c}
\tau_{H L}+\tau_{H R} \\
0 \\
\tau_{V F L}+\tau_{V F R}+\tau_{V B L}+\tau_{V B R} \\
0 \\
0 \\
\tau_{H L} \cdot l_{L}-\tau_{H R} \cdot l_{R}
\end{array}\right)=\left(\begin{array}{c}
F_{u} \\
0 \\
F_{w} \\
0 \\
0 \\
M_{r}
\end{array}\right)
$$

where $\tau_{i}$ is the force delivered by thruster $i$. The subscripts $H L$ and $H R$ stand for horizontal left (port side) and horizontal right (starboard). $V F L$ vertical front left, $V F R$ vertical front right, $V B L$ vertical back left, and $V B R$ vertical back right, respectively. $l_{L}, l_{R}$ are the distances between the center of gravity and the locations of the two horizontal thrusters.

Substituting these matrices and vectors into (1), the decoupled model for the Canterbury UV is

$$
\begin{aligned}
\left(m-X_{\dot{u}}\right) \dot{u} & =-X_{u} u-X_{u|u|} u|u|+F_{u} \\
\left(m-Z_{\dot{w}}\right) \dot{w} & =-Z_{w} w-Z_{w|w|} w|w|+F_{w}+(W-B) \\
\left(I_{z}-N_{\dot{r}}\right) \dot{r} & =-N_{r} r-N_{r|r|} r|r|+M_{r}
\end{aligned}
$$

\section{EXTENDED KALMAN FILTER BASED POSITION ESTIMATION}

One key problem of a UV is the determination of its position. Because the vehicle cannot receive a GPS signal when it is submerged, the position has to be estimated. This can be done using the measurement data of the vehicle's acceleration and velocity. The most used estimator in the area of autonomous navigation is a Kalman filter Drolet et al. (2000). As the Canterbury UV dynamics is nonlinear, the extended Kalman filter (EKF) was used instead of the Kalman filter which is specifically suitable for linear applications.

As the degrees of freedom are decoupled, the filter is designed for the movement in one direction, the surge direction. The design of EKF for the other directions is similar. It is assumed that both acceleration and velocity are measured by onboard sensors.

For EKF, the state equation can be written as

$$
\underbrace{\left(\begin{array}{c}
\psi_{k+1} \\
\dot{\psi}_{k+1} \\
x_{k+1} \\
y_{k+1} \\
u_{k+1} \\
\dot{u}_{k+1}
\end{array}\right)}_{\mathbf{x}_{k+1}}=\underbrace{\left(\begin{array}{c}
\psi_{k}+\Delta t \dot{\psi}_{k}+w_{\psi_{k}} \\
\dot{\psi}_{k}+w_{\dot{\psi}_{k}} \\
x_{k}+\Delta t \cos \psi_{k} u_{k}+\frac{1}{2} \Delta t^{2} \cos \psi_{k} \dot{u}_{k}+w_{x_{k}} \\
y_{k}+\Delta t \sin \psi_{k} u_{k}+\frac{1}{2} \Delta t^{2} \sin \psi_{k} \dot{u}_{k}+w_{y_{k}} \\
u_{k}+\Delta t \dot{u}_{k}+w_{u_{k}} \\
\dot{u}_{k}+w_{\dot{u}_{k}}
\end{array}\right)}_{f\left(\mathbf{x}_{k}, \mathbf{u}_{k}, \mathbf{w}_{k}\right)}
$$

where $\Delta t$ denotes the sampling period of the sensors.

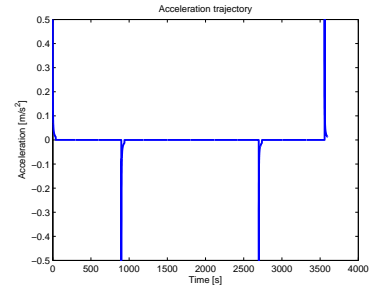

Fig. 3. Acceleration trajectory used for simulation

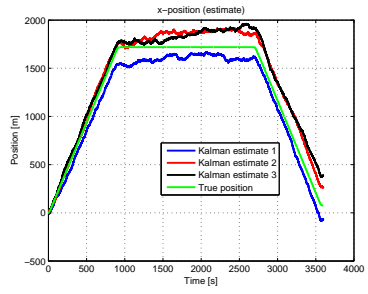

(a) With acceleration \& velocity

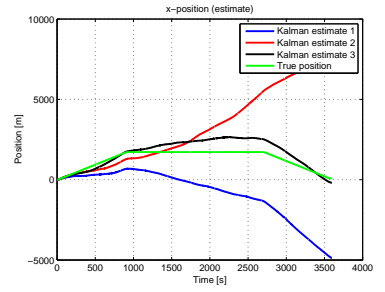

(b) With acceleration only
Fig. 4. Estimated position of the vehicle

The measurement equation can be written as

$$
\underbrace{\left(\begin{array}{l}
z_{1_{k}} \\
z_{2_{k}} \\
z_{3_{k}} \\
z_{4_{k}}
\end{array}\right)}_{\mathbf{z}_{k}}=\underbrace{\left(\begin{array}{llllll}
1 & 0 & 0 & 0 & 0 & 0 \\
0 & 1 & 0 & 0 & 0 & 0 \\
0 & 0 & 0 & 0 & 1 & 0 \\
0 & 0 & 0 & 0 & 0 & 1
\end{array}\right)}_{\mathbf{H}} \underbrace{\left(\begin{array}{l}
\psi_{k} \\
\dot{\psi}_{k} \\
x_{k} \\
y_{k} \\
u_{k} \\
\dot{u}_{k}
\end{array}\right)}_{\mathbf{x}_{k}}+\underbrace{\left(\begin{array}{l}
v_{1_{k}} \\
v_{2_{k}} \\
v_{3_{k}} \\
v_{4_{k}}
\end{array}\right)}_{\mathbf{v}_{k}}
$$

Take an IMU (3DM-GX1, MicroStrain, VT) for example, it has an accuracy for the yaw angle measurement of $2.8^{\circ}=0.0489 \mathrm{rad}$, an accuracy for the angular rate of $2.3^{\circ} / \mathrm{sec}=0.0401 \mathrm{rad} / \mathrm{sec}$ and a noise on the acceleration measurement of $0.1 \mathrm{~m} / \mathrm{s}^{2}$. Assume the velocity sensor have an accuracy of $1 \mathrm{~m} / \mathrm{s}$, so the measurement noise covariance matrix can be

$$
\begin{aligned}
\mathbf{R} & =\operatorname{diag}\left(\sigma_{I M U, \psi}^{2}, \sigma_{I M U, \dot{\psi}}^{2}, \sigma_{S E N S, u}^{2}, \sigma_{I M U, \dot{u}}^{2}\right) \\
& =\operatorname{diag}\left(0.0489^{2}, 0.0401^{2}, 1^{2}, 0.1^{2}\right) .
\end{aligned}
$$

The process noise is assumed to be zero mean white noise and independent. The covariance matrix $\mathbf{Q}$ of the process noise is given by

$$
\mathbf{Q}=\operatorname{diag}\left(\sigma_{\psi}^{2}, \sigma_{\dot{\psi}}^{2}, \sigma_{x}^{2}, \sigma_{y}^{2}, \sigma_{u}^{2}, \sigma_{\dot{u}}^{2}\right) .
$$

It has to be tuned during experiments. But in simulation we can choose $\mathbf{Q}=\operatorname{diag}\left(10^{-3}, 10^{-3}, 10^{-3}, 10^{-3}, 10^{-3}, 10^{-5}\right)$. The initial estimates are $\hat{\mathbf{x}}_{0 \mid 0}=[0,0,0,0,0, .5]^{T}$ and $\mathbf{P}_{0 \mid 0}=\mathbf{I}$.

As the vehicle reaches a constant velocity after a few seconds, the acceleration trajectory shown in Fig. 3 was used for simulation. With this (noise free) acceleration the 'true' velocity and the position of the vehicle can be calculated, and used for a comparison with the vehicle's position estimated from EKF. Three sets of simulated noisecontaminated measurements were formed and filtered with EKF. The position estimate is shown in Fig. 4(a). 
A vehicle solely relying on the acceleration will have a larger error on navigation. A simulation assuming the velocity is unknown was conducted for the same position estimation problem is shown in Fig. 4(b), as a comparison.

Even with both acceleration and velocity measured, the position estimated in Fig. 4(a) still contains an error due to the fact that the position is never calibrated. This can be corrected by surfacing the vehicle intermittently to calibrate its position via GPS Stutters et al. (2008). This was validated in simulation (data not shown).

\section{NAVIGATION CONTROL}

This section will simulate the navigation control based on the dynamic equations established in Section 2.8. Again, because the degrees of freedom are decoupled, simulations are limited to $2 D$ trajectories, e.g. the heave direction is not taken into account.

Because the parameters of the Canterbury UV are unknown (except for the thruster positions) at this time, the values are taken from Smallwood and Whitcomb (2004), where the vehicle is comparable to ours. It is known that the Canterbury vehicle reaches its maximum velocity after about four seconds of full thrust. With this information, the parameters are slightly tuned to fit our model and are shown in table 2 .

\subsection{Lyapunov Stability}

Combining (22) and (24) yields

$$
\begin{array}{r}
\left(\begin{array}{cc}
m-X_{\dot{u}} & 0 \\
0 & I_{z}-N_{\dot{r}}
\end{array}\right)\left(\begin{array}{l}
\dot{u} \\
\dot{r}
\end{array}\right)= \\
\left(\begin{array}{c}
-X_{u} u-X_{u|u|} u|u| \\
-N_{r} r-N_{r|r|} r|r|
\end{array}\right)+\underbrace{\left(\begin{array}{cc}
1 & 1 \\
l_{L}-l_{R}
\end{array}\right)}_{\mathbf{C}} \underbrace{\left(\begin{array}{c}
F_{H L} \\
F_{H R}
\end{array}\right)}_{\mathbf{u}}
\end{array}
$$

or

$$
\left(\begin{array}{c}
\dot{u} \\
\dot{r}
\end{array}\right)=\left(\begin{array}{l}
\frac{-X_{u}}{m-X_{\dot{u}}} u-\frac{X_{u|u|}}{m-X_{\dot{u}}} u|u| \\
\frac{-N_{r}}{I_{z}-N_{\dot{r}}} r-\frac{N_{r|r|}}{I_{z}-N_{\dot{r}}} r|r|
\end{array}\right)+\mathbf{C u}=\left(\begin{array}{l}
f_{1}(u) \\
f_{2}(r)
\end{array}\right)+\mathbf{C u} \text { (30) }
$$

For the first term on the right hand side, the Taylor expansion can be applied around the fixed point $\left(\left(u_{0}, r_{0}\right)\right.$, such that $f_{1}\left(u_{0}\right)=0$ and $f_{2}\left(r_{0}\right)=0$, solved to be $\left.(0,0)\right)$ to linearize the dynamics. Substituting values in table 2 into the Taylor expansion of (30) yields

$$
\begin{aligned}
& \underbrace{\left(\begin{array}{c}
\dot{u} \\
\dot{r}
\end{array}\right)}_{\dot{\mathbf{x}}} \approx\left(\begin{array}{cc}
\frac{\mathrm{d} f_{1}}{\mathrm{~d} u}\left(u_{0}\right) & 0 \\
0 & \frac{\mathrm{d} f_{2}}{\mathrm{~d} r}\left(r_{0}\right)
\end{array}\right)\left(\begin{array}{l}
u \\
r
\end{array}\right)+\mathbf{C u} \\
& =\underbrace{\left(\begin{array}{cc}
-0.399 & 0 \\
0 & -0.410
\end{array}\right)}_{\mathbf{F}} \underbrace{\left(\begin{array}{l}
u \\
r
\end{array}\right)}_{\mathbf{x}}+\mathbf{C u}
\end{aligned}
$$

For Lyapunov equation $\mathbf{F}^{T} \mathbf{P}+\mathbf{P F}=-\mathbf{Q}$, as the eigenvalues of the $\mathbf{F}$ matrix are $<0$, choosing $\mathbf{Q}=\mathbf{I}$, a positive define matrix $\mathbf{P}=\operatorname{diag}(1.253,1.219)$.

So the derivative of the Lyapunov function becomes
Table 2. Parameter values used for simulation

$$
\begin{array}{cll}
\text { Parameter } & \text { Value } & \text { Unit } \\
\hline m-X_{\dot{u}} & 150.3 & {[\mathrm{~kg}]} \\
X_{u} & 60 & {[\mathrm{~kg} / \mathrm{s}]} \\
X_{u|u|} & 173.4 & {[\mathrm{~kg} / \mathrm{m}]} \\
I_{z}-N_{\dot{r}} & 98.04 & {\left[\mathrm{~kg} \cdot \mathrm{m}^{2} / \mathrm{rad}\right]} \\
N_{r} & 40.2 & {\left[\mathrm{~kg} \cdot \mathrm{m}^{2} / \mathrm{rad} \cdot \mathrm{s}\right]} \\
N_{r|r|} & 187.1 & {\left[\mathrm{~kg} \cdot \mathrm{m}^{2} / \mathrm{rad}^{2}\right]} \\
l_{L}=l_{R} & 0.4 & {[\mathrm{~m}]} \\
\hline \multicolumn{4}{|c}{} \\
\dot{V}(\mathbf{x})=-\mathbf{x}^{T} \mathbf{Q} \mathbf{x}+2 \mathbf{x}^{T} \mathbf{P g}(\mathbf{x}) \\
=-\left(u^{2}+r^{2}\right)-2.892 u^{3} \operatorname{sgn}(u)-4.652 r^{3} \operatorname{sgn}(r)
\end{array}
$$

From (33) it is clear that $\dot{V}(\mathbf{x}) \leq 0$, thus making the system Lyapunov stable.

\subsection{Bounded Input Bounded Output}

For systems $\dot{\mathbf{x}}=\mathbf{A} \mathbf{x}+\mathbf{B u}$ to be stable in terms of bounded input bounded output, the real parts of the eigenvalues of A should $<0$.

Linearizing the system described in (30) about any state $\left(u^{*}, r^{*}\right)$ results in

$$
\dot{\mathbf{x}}=\underbrace{\left(\begin{array}{cc}
-0.399-2.308\left|u^{*}\right| & 0 \\
0 & 0.410-3.816\left|r^{*}\right|
\end{array}\right)}_{\mathbf{A}} \times \underbrace{\left(\begin{array}{c}
f_{1}\left(u^{*}\right) \\
f_{2}\left(r^{*}\right)
\end{array}\right)}_{\text {constant }}+\mathbf{C u}(34)
$$

The eigenvalues of the $\mathbf{A}$ matrix are always $<0$, making the system asymptotically stable for all bounded inputs.

\subsection{Navigation Control Simulation}

Assume the vehicle is required to follow a specified trajectory. This section shows the simulation result using the dynamics established in Section 2.8. The EKF described in Section 3 is integrated to estimate vehicle position. Here, the reference signal is a $2 D$-trajectory which consists of $(x, y)$ coordinates. For the vehicle to follow this trajectory, three signals are fed back to the controller, namely the $x$-position, $y$-position and the yaw angle. With the $x$ and $y$-position, the position error is calculated. With this position error, the desired yaw angle is calculated using $\psi_{d}=\arctan \left(\frac{y_{t}-y_{c}}{x_{t}-x_{c}}\right)$ to maintain the heading of the vehicle.

Using the waypoint guidance by line-of-sight Healey and Lienard (1993), we determine the thruster input with a PI-controller for the position and a P-controller for the yaw angle. A PI-controller, not requiring the UV dynamics Sun and Cheah (2003), makes the control robust. The integrator will lead the final error to zero. A P-controller is used for the yaw angle because the final error for the yaw angle does not need to go to zero. Although a P(I)controller is linear and the Canterbury UV has non-linear dynamics, the performance remains satisfying. This can be seen in Fig. 5, where the UV tries to follow an ellipse five times. The UV starts from the center of the ellipse, with zero initial yaw angle. The EKF enables the UV to follow the predefined trajectory more accurately.

\section{DISCUSSIONS AND FUTURE WORK}

Using EKF brings favorable advantages: less control cost, less overshoot, and faster settling. This could be illustrated 


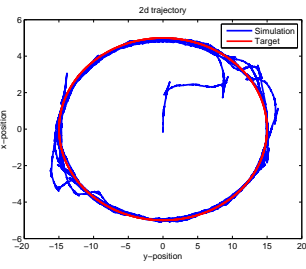

(a) Without EKF

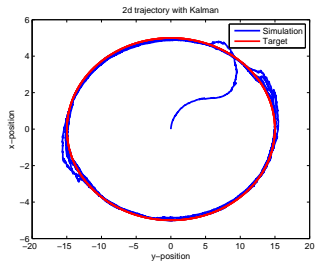

(b) With EKF
Fig. 5. 2D-position using circular trajectory

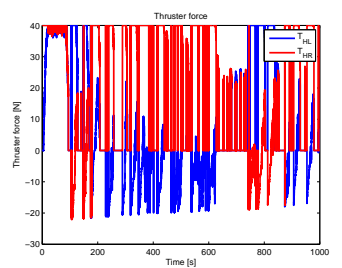

(a) Without EKF

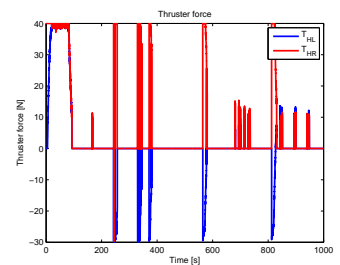

(b) With EKF
Fig. 6. Control signal for thrusters in a $2 \mathrm{D}$ single point trajectory

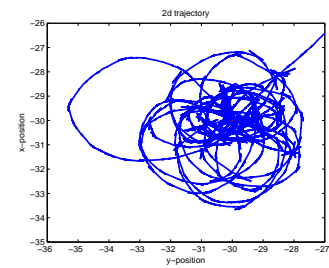

(a) Without EKF

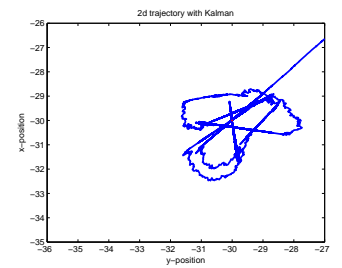

(b) With EKF
Fig. 7. Real path of the vehicle in a $2 \mathrm{D}$ single point trajectory

by another example. Suppose the vehicle moves from the origin to point $(-30,-30)$ with zero initial yaw angle. As shown in Fig. 6, without EKF, the measurement noise causes position drift from the target and the vehicle keeps correcting by exciting the thrusters; while with EKF, the thrusters are much less excited, and thus consume less power, which is favored for the limited onboard power supply. Fig. 7 shows the $2 D$ motion path of the vehicle, verifying EKF's effectiveness: the vehicle stays closer to the target and does not correct its position as often.

In future, the parameters of the Canterbury UV given in table 2 can be determined experimentally. Furthermore, the model needs to be validated by sea trial experiments and fine-tuned by including environmental disturbances, such as ocean currents. Computer vision is also considered to provide an extra feedback for navigation.

\section{CONCLUSIONS}

The hover-capable Canterbury UV is modeled and simulated. Due to its special features, such as symmetric w.r.t. the $x-z$ plane and close to symmetric w.r.t. the $y-z$ plane, low-speed, stable in the roll and pitch angle, the motions in the six degrees of freedom can be decoupled and controlled individually by the thrusters. The non-linear model of the vehicle is proven to be stable. Using the extended Kalman filter results in better control performance at lower cost for navigation. The current model, to be validated and fine-tuned by experiments in future, can provide a sound starting platform to design and optimize navigation controllers for the Canterbury UV.

\section{REFERENCES}

Alessandri, A., Caccia, M., Indiveri, G., and Veruggio, G. (1998). Application of LS and EKF techniques to the identification of underwater vehicles. Proc 1998 IEEE International Conference on Control Applications, 1084-1088.

Antonelli, G. (2007). On the use of adaptive/integral actions for six-degrees-of-freedom control of autonomous underwater vehicles. IEEE Journal of Oceanic Engineering, 32(2), 300-312.

Drolet, L., Michaud, F., and Côté, J. (2000). Adaptable sensor fusion using multiple Kalman filters. Proc 2000 IEEE/RSJ International Conference on Intelligent Robots and Systems, 2, 1434-1439.

El-Hawary, F. (2001). The ocean engineering handbook. CRC Press LLC.

Fossen, T. (1994). Guidance and control of ocean vehicles. John Wiley and Sons Ltd., New York, second edition.

Griffiths, G. (2003). Technology and applications of autonomous underwater vehicles. Taylor \& Francis, New York.

Healey, A. and Lienard, D. (1993). Multivariable sliding mode control for autonomous diving and steering of unmanned underwater vehicles. IEEE Journal of Oceanic Engineering, 18, 327-339.

Jones, D., Clarke, D., Brayshaw, I., Barillon, J., and Anderson, B. (2002). The calculation of hydrodynamic coefficients for underwater vehicles. DSTO Platforms Sciences Laboratory.

Ridao, P., Tiano, A., El-Fakdi, A., Carreras, M., and Zirilli, A. (2004). On the identification of non-linear models of unmanned underwater vehicles. Control Engineering Practice, 12, 1483-1499.

Silpa-Anan, C., Abdallah, S., and Wettergreen, D. (2000). Development of autonomous underwater vehicle towards visual servo control. Proc Australian Conference on Robotics and Automation, 105-110.

Smallwood, D. and Whitcomb, L. (2004). Model-based dynamic positioning of underwater robotic vehicles: theory and experiment. IEEE Journal of Oceanic Engineering, 29(1), 169-186.

Stutters, L., Liu, H., Tiltman, C., and Brown, D. (2008). Navigation technologies for autonomous underwater vehicles. IEEE Trans on Systems, Man and Cybernetics, Part C: Applications and Reviews, 38(4), 581-589.

Sun, Y. and Cheah, C. (2003). Adaptive setpoint control for autonomous underwater vehicles. Proc 42nd IEEE Conference on Decision and Control, 1262-1267.

van de Ven, P., Flanagan, C., and Toal, D. (2005). Neural network control of underwater vehicles. Engineering Applications of Artificial Intelligence, 18, 533-547.

Wang, W. and Clark, C. (2006). Modeling and simulation of the VideoRay Pro III underwater vehicle. OCEANS 2006 - Asia Pacific, 1-7.

Wang, W., Chen, X., Marburg, A., Chase, J., and Hann, C. (2008). A low-cost unmanned underwater vehicle prototype for shallow water tasks. Proc 2008 IEEE/ASME MESA, 204-209. 